Received: 26 January 2017

Accepted: 12 May 2017

Published online: 21 June 2017
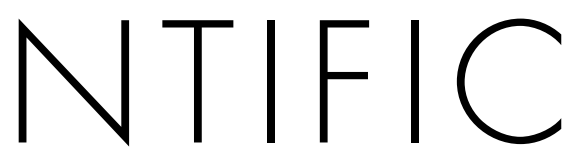

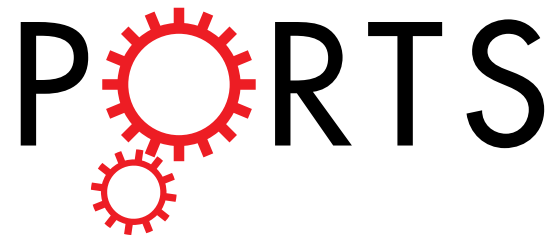

\title{
OPEN Voxel-based morphometric brain comparison between healthy subjects and major depressive disorder patients in Japanese with the s/s genotype of 5-HTTLPR
}

Natsuki Igata ${ }^{1}$, Shingo Kakeda ${ }^{1}$, Keita Watanabe ${ }^{1}$, Satoru Ide ${ }^{1}$, Taro Kishi ${ }^{3}$, Osamu Abe ${ }^{4}$, Ryouhei Igata ${ }^{2}$, Asuka Katsuki ${ }^{2}$, Nakao Iwata ${ }^{3}$, Reiji Yoshimura ${ }^{2}$ \& Yukunori Korogi ${ }^{1}$

Individuals with s/s genotype of serotonin transporter gene-linked promotor region (5-HTTLPR), which appear with a high frequency in Japanese, exhibit more diagnosable depression in relation to stressful life events than those with the $s / /$ or $/ / l$ genotype. We prospectively investigated the brain volume changes in first-episode and medication naïve major depression disorder patients (MDD) with the s/s genotype in Japanese. We assessed the differences between 27 MDD with the s/s genotype and $\mathbf{4}$ healthy subjects (HS) with the same genotype using a whole-brain voxel-by-voxel statistical analysis of MRI. Gray matter volume in a brain region with significant clusters obtained via voxel-based morphometry analysis were measured and, as an exploratory analysis, evaluated for relationships to the subcategory scores (core, sleep, activity, psychic, somatic anxiety, delusion) of the Hamilton Depression Rating Scale (HAM-D) and the Social Readjustment Rating Scale (SRRS). The brain volume in the left insula lobe was significantly smaller in the MDD than in the HS. The left insula lobe volume correlated negatively with the "psychic" score of HAM-D and the SRRS. In a Japanese population with the s/s genotype, we found an atrophy of the insula in the MDD, which might be associated with "psychic" symptom and stress events.

One of the major genetic factors determining inter-individual differences in stress reactivity is the serotonin transporter (5-HTT) gene polymorphism, which mediates reuptake and recycling of released serotonin following neuronal stimulation. Neurotransmission mediated by serotonin $(5-\mathrm{HT})$ contributes to many physiological functions, such as motor activity, food intake, sleep, and emotional states ${ }^{1,2}$. There are two common alleles: a 44-bp insertion (l-allele) or deletion (s-allele). Transcriptional activity of the l-allele of the gene is twice that of the s-allele $e^{3}$. Thus, the s promoter allelic variant is linked to reduced 5-HTT mRNA expression, resulting in less serotonin reuptake than with the l-allelic variant ${ }^{4,5}$.

Many studies have suggested an association between the 5-HTT genotype and physiological reactivity to acute stressors $^{6,7}$. Gotlib et al. found that individuals with the s/s genotype showed enhanced cortisol secretion in response to acute psychological stressors ${ }^{7}$. The most recent meta-analysis regarding the 5-HTT gene-linked polymorphic region (5-HTTLPR) confirmed the robust link between the s/s genotype of the 5-HTTLPR, the experience of stress, and resultant depression ${ }^{8}$. Therefore, individuals with the s/s genotype are more sensitive to the depressogenic effects of all stressful life events than those with the $\mathrm{s} / \mathrm{l}$ or $1 / 1$ genotype.

For the 5-HTT genotype, the distribution of alleles in Caucasians is significantly different from that in the Japanese population. Lesch et al. reported that a PCR-based genotype analysis in Caucasian subjects revealed allele frequencies of $32 \%$ for the $1 / 1$ genotype, $49 \%$ for the $1 / \mathrm{s}$, and $19 \%$ for the $\mathrm{s} / \mathrm{s}^{4}$. In contrast, in 501 Japanese

${ }^{1}$ Department of Radiology, University of Occupational and Environmental Health, Kitakyushu, Japan. ${ }^{2}$ Department of Psychiatry, University of Occupational and Environmental Health, Kitakyushu, Japan. ${ }^{3}$ Department of Psychiatry, Fujita Health University, School of Medicine, Toyoake, Japan. ${ }^{4}$ Department of Radiology, Graduate School of Medicine, University of Tokyo, Tokyo, Japan. Correspondence and requests for materials should be addressed to S.K. (email: kakeda@med.voeh-u.ac.jp) 
healthy subjects, the frequency distribution of the $1 / 1,1 / s$, and $s / s$ genotypes were $3 \%(n=16), 32 \%(n=159)$, and $65 \%(n=326)$, respectively 9 . Therefore, because differences in racial genetic backgrounds may be contributing factors, additional assessments regarding the 5-HTT genotype must be conducted separately for each race group.

Major Depressive Disorder (MDD) is not a homogeneous disease. For example, Parker has proposed that depression may have various forms ${ }^{10}$, and Ostergaard and colleagues ${ }^{11}$ pointed out that MDD may present in 1497 different combinations of the symptoms that are used to define it ${ }^{12}$. Recent data which demonstrate the different neurobiological underpinnings, pathways and required treatments for at least four different "subtypes" of depression based upon separation of MDD symptoms into distinct categories according to symptom coherence ${ }^{13,14}$. In other words, MDD is a heterogeneous disorder, which origins from various pathophysiology. We focused MDD dimensions according to the components proposed by Seretti et al. ${ }^{15}$, in which Hamilton Depression Rating Scale (HAM-D) items were grouped into 6 factors (core, sleep, activity, psychic, somatic anxiety, and delusion).

Morphological brain abnormalities in MDD patients may be attributable to genetic- and epigenetic factors that regulate brain development and neurodegeneration. In MDD, many previous magnetic resonance imaging (MRI) studies using the voxel-based morphometry (VBM) technique have reported evidence of a relationship between brain volume and genetic factors, including brain-derived neurotrophic factor (BDNF), norepinephrine transporter (NET) gene (SLC6A2), and methylenetetrahydrofolate reductase (MTHFR)/catechol-O-methyltransferase (COMT) polymorphisms ${ }^{16-22}$. To our knowledge, however, there has only been one VBM study of MDD patients and HS regarding the association between the triallelic polymorphism in 5-HTTLPR and gray matter (GM) brain volume. The authors assessed the effect of 5-HTTLPR polymorphisms by comparing l-allele individuals versus s-allele ones (the 1/s genotype as well as the s/s genotype) because of the limited number of s/s genotype individuals available. Thus, the morphological brain changes in MDD patients with the s/s genotype of 5-HTTLPR have not been fully evaluated. We therefore examined the brain volume changes in first-episode and medication naïve MDD patients with the s/s genotype of 5-HTTLPR using 3-Tesla MRI data from a Japanese population.

\section{Materials and Methods}

Study Participants. Human experiments were carried out in accordance to guidelines provided and approved by the Institutional Review Board of University of Occupational and Environmental Health School of Medicine, Japan (approval number: H25-13). The protocol of this prospective study was approved by the Ethics Committee of the University of Occupational and Environmental Health. All of the participants provided their written informed consent to participate in the study. Twenty-seven first-episode treatment-naïve MDD patients were recruited. All patients were consistent right-handers. A majority of subjects in this study participated in an earlier published study, which analyzed the relationship of brain volume to the catechol-O-methyl transferase $(\mathrm{COMT})^{21}$, brain-derived neurotrophic factor $(\mathrm{BDNF})^{19}$, and norepinephrine transporter $(\mathrm{NET})^{22}$ gene polymorphism. A psychiatrist (K.H. with seven years of experience in psychiatry) diagnosed a major depressive episode using the Structured Clinical Interview according to the Diagnostic and Statistical Manual of Mental Disorders (DSM)-IV-TR criteria. The severity of depression was evaluated using the17-item HAM-D. The exclusion criteria were a history of neurological diseases or other physical diseases and the presence of comorbidities with other disorders (no evidence of schizoaffective disorder, bipolar disorder, Axis II, personality disorders, or mental retardation).

Forty-seven healthy subjects (HS) were also recruited via an interview conducted by the same psychiatrist using the Structured Clinical Interview for DSM-IV, non patient edition. None of them had a history of serious medical or neuropsychiatric illness or a family history of major psychiatric or neurological illness among their first-degree relatives.

Genotyping. Seventy-four subjects from the neuroimaging study provided a blood sample, from which DNA was extracted using standard laboratory protocols. DNA was isolated from peripheral blood mononuclearcells using the QIAamp DNA Mini-Kit (QIAGEN, Tokyo, Japan). Genotyping was carried out with a polymerase chain reaction (PCR) SNP genotyping system using the BigDye Terminator v3.1 Cycle Sequencing Kit (Life Technologies Japan, Tokyo, Japan). The DNA was read using a BMG Applied Biosystem 3730xI DNA Analyzer (Life Technologies Japan). We used a forward primer (5'-GGC GTT GCC GCT CTG AAT GC-3') and a reverse primer (5'-GAG GGA CTG AGC TGG ACA ACC AC-3') for the 5-HTTLPR polymorphism. The genotyping revealed that all $27 \mathrm{MDD}$ patients had the s/s genotype of 5-HTTLPR. Among $47 \mathrm{HS}, 3 \mathrm{had}$ l/s, and 44 had s/s genotypes of 5-HTTLPR. Therefore, the $44 \mathrm{HS}$ with the s/s genotype of 5-HTTLPR were assessed in this study.

MRI and Image Processing for Voxel-Based Morphometry (VBM). All MDD patients underwent brain MRI before receiving antidepressant medication or psychotherapy. Therefore, all participants were medication naïve at the time of MRI. MRI data were obtained using a 3.0-Tesla scanner (Signa EXCITE 3 T; GE Medical Systems, Milwaukee, WI, USA) with a 3-dimensional fast-spoiled gradient-recalled acquisition with steady state (3D-FSPGR). The following parameters were used: repetition time ms/echo time ms/inversion time, 10/4.1/700; flip angle, 10; field of view, $24 \mathrm{~cm}$; section thickness, $1.2 \mathrm{~mm}$; and resolution, $0.9 \times 0.9 \times 1.2 \mathrm{~mm}$. All of the images were corrected for image distortion due to gradient non-linearity using "GradWarp" ${ }^{23}$ and for intensity inhomogeneity using "N3"24. The SPM8 software program was used for the image processing of VBM.

The 3D-FSPGR images in native spaces were spatially normalized and segmented into GM, white matter, and cerebrospinal fluid images, and were intensity-1modulated using the Diffeomorphic Anatomical Registration Through Exponential Lie Algebra (DARTEL) toolbox in SPM8 ${ }^{25}$. DARTEL was proposed by Ashburner as an alternative method for normalization in the SPM package ${ }^{26}$. For intensity modulation, the voxel values of the segmented images were multiplied by the measure of the warped and unwarped structures derived from the nonlinear step of the spatial normalization. This step converted the relative regional GM density into absolute 


\begin{tabular}{|l|l|l|l|}
\hline & HS (n=44) & MDD $(\mathbf{n}=\mathbf{2 7})$ & $\mathbf{p}$ \\
\hline Age, mean (SD) & $41.2(11.6)$ & $45.8(12.7)$ & 0.13 \\
\hline Female, numbers & 12 & 12 & 0.14 \\
\hline $\begin{array}{l}\text { Total gray matter volume, } \\
\text { mean (SD) (ml) }\end{array}$ & $698.7(63.0)$ & $665.6(65.7)$ & 0.04 \\
\hline $\begin{array}{l}\text { HAMD17, mean of total } \\
\text { scores (SD) }\end{array}$ & & $21.8(4.71)$ & \\
\hline
\end{tabular}

Table 1. Demographic and clinical characteristics of participants. Abbreviations: HS=healthy subjects; $\mathrm{MDD}=$ Major depression disorders; HAMD = 17-item Hamilton Rating Scale for Depression

GM density, expressed as the amount of GM per unit volume of brain tissue before spatial normalization. The resulting modulated GM and white matter images were smoothed with an 8-mm Gaussian kernel.

Hamilton Depression Rating Scale and Social Readjustment Rating Scale. To evaluate the specific subcategories of depressive symptoms, the HAM-D items were grouped according to the following factors: core (items 1, 2, 7, 8, 10, and 13), sleep (items 4.6), activity (items 7 and 8), psychic (items 9 and 10), somatic anxiety (items 11.13), and delusion (items 2, 15, and 20), as described by Serretti et al..$^{15}$. In addition, stressful life events were assessed using the Social Readjustment Rating Scale (SRRS), which is a major life events inventory developed by Holmes and Rahe ${ }^{27}$. The SRRS assesses frequency of common stressful life events that occurred over the previous 12 months. The SRRS has been widely used in studies of psychosocial stress and illness, and includes 43 stressful life events, each scored from 0 to 100 units of life change (ULC).

Statistical Analysis. For the analysis of demographic and clinical characteristics of participants, a multivariate analysis of variance was performed to compare the differences in age and total GM volume between HS and MDD patients. In the VBM analysis, statistical analyses were performed using the SPM8 software program. The morphological changes in the GM were assessed using a two-sample t-test with diagnosis status. Age, sex, and total GM volume were included as covariates of no interest into all analyses with HS as confounding variables. The differences in the GM volume between MDD patients and HS with the s/s genotype were assessed at the whole-brain level. This analysis yielded statistical parametric maps (SPMs $[t]$ ) based on a voxel-level height threshold of $\mathrm{p}<0.001$. We used cluster level family wise error (FWE) correction. The significance level was set at an FWE-corrected $\mathrm{p}<0.05$. GM volume in a brain region with significant clusters obtained on the VBM analysis were measured by using region of interest (ROI) analyses and, as an exploratory analysis, evaluated for relationships to the subcategory scores of HAM-D and the SRRS using Spearman's rank correlation.

The statistical analyses were performed using the statistical software package StatView 5.0 (SAS Institute, Cary, NC, USA). A p-value of $<0.05$ was assumed to indicate a statistically significant difference, except for with the SPM8 analysis. Using Spearman's rank correlation, we assessed the relationship between the subcategory scores (core: $0-22$, sleep: $0-4$, activity: $0-8$, psychic: $0-8$, somatic anxiety: $0-6$, and delusion: $0-8$ ) of the Hamilton Depression Rating Scale (HAM-D) and the GM volume in a brain region with significant clusters obtained on the VBM analysis.

\section{Results}

Demographic and Clinical Data. There was no significant difference between the two groups in the distribution of sex and age (Table 1). There was a significant difference between the two groups in the total GM volume, with the volume lower in MDD patients than in HS $(\mathrm{p}<0.05)$.

Whole-brain VBM Analysis (MDD vs. HS with the s/s genotype of 5-HTTLPR).

The whole-brain VBM analysis demonstrated that the volume of the left insula lobe was significantly smaller for the MDD patients than for the HS (FWE-corrected $\mathrm{p}=0.049$ ) (Fig. 1, Table 2). In other regions, we found no significant differences of the brain volume between the MDD patients and the HS.

ROI analysis of significant clusters. We obtained the subcategory scores of HAM-D from all 27 MDD patients. There was no relationship between total HAM-D score and the volume of the left insula lobe. We assessed relationship between the subcategory scores of HAM-D and the volume of the left insula lobe with significant clusters in the VBM analysis. Our exploratory analysis showed a significant correlation between the "psychic (items 9 and 10)" score of the HAM-D and the volume of the left insula lobe (Spearman rank correlation: uncorrected-p $=0.04, \mathrm{R}=-0.40$ ) (Fig. 2, Table 3). We found no relationship between other subcategory scores and the volume of the left insula lobe.

We obtained data of SRRS from 26 of 27 MDD patients. For relationship between the SRRS and the volume of the left insula lobe, an inverse tendency was observed (Spearman rank correlation: uncorrected- $\mathrm{p}=0.05$, $\mathrm{R}=-0.39$ ) (Fig. 3).

\section{Discussion}

Previous studies have shown difference in the allele frequency between Japanese and Caucasian populations, with a low frequency of the l-allele in Japanese populations ${ }^{9}$. In the current study, there were no MDD patients with the $1 / \mathrm{s}$ or $1 / 1$ genotype of 5-HTTLPR, although the reason for this predominance of s/s is unclear. Therefore, we assessed the differences in the GM volume between the MDD patients with the s/s genotype of 5-HTTLPR and the HS with the same genotype. We found that the volume of the left insula lobe was significantly smaller in the MDD patients than in the HS. Of note, there was a significant negative correlation between the volume of the left 

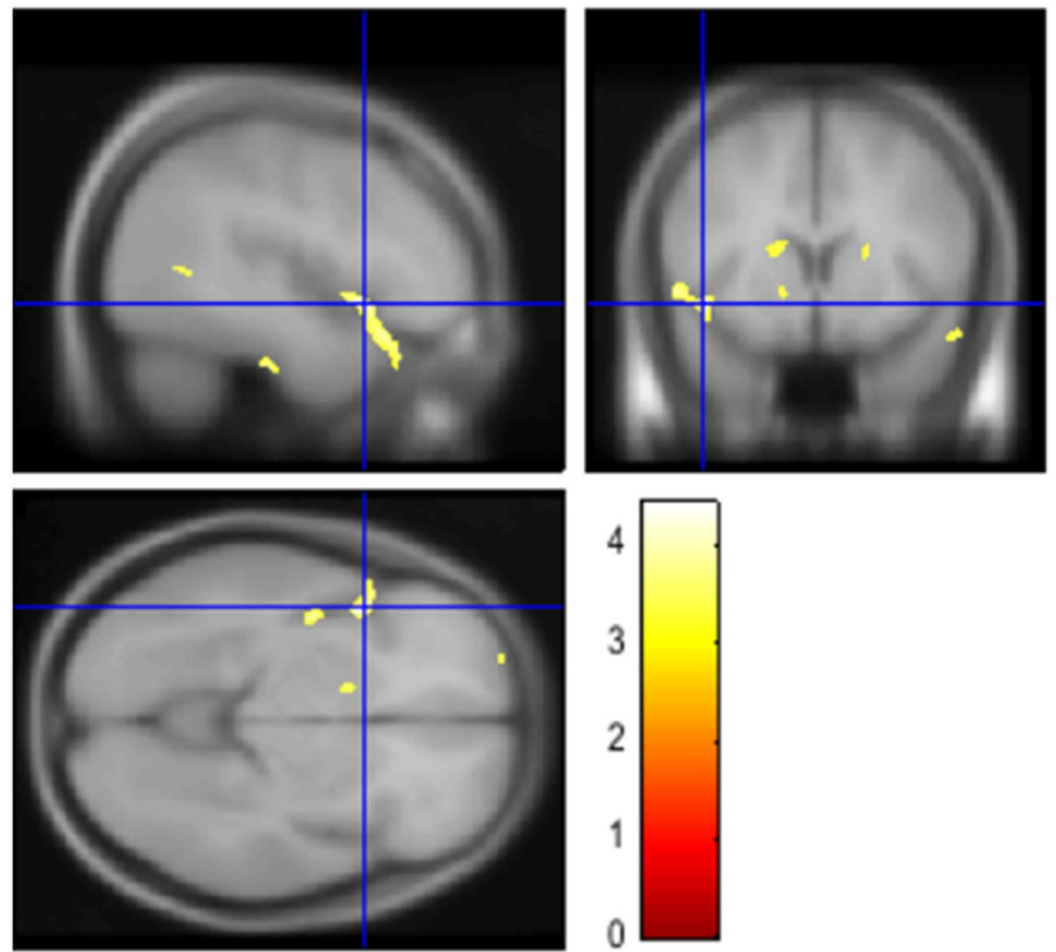

Figure 1. The diagnosis effects (MDD vs. HS with the s/s genotype of 5-HTTLPR). The SPM ${ }^{45}$ is displayed on the T1 weighted MRI. The volume of the left insula lobe was smaller in MDD patients than in HS (FWEcorrected $\mathrm{p}=0.049, \mathrm{~T}=4.40)$. The color bar represents the range of the $T$-value.

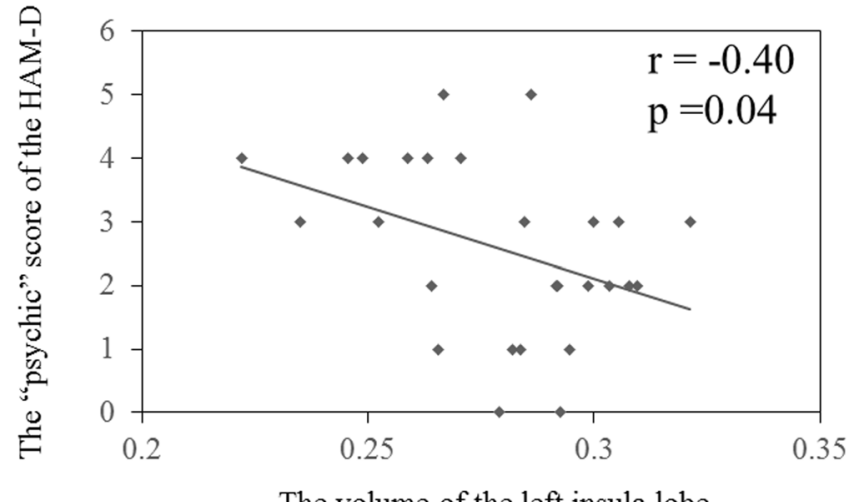

The volume of the left insula lobe

Figure 2. Relationship between the "psychic (items 9 and 10)" score of the HAM-D and the volume of the left insula lobe. The higher "psychic (items 9 and 10)" score of the HAM-D is associated with the volume reduction of the left insula lobe (Spearman rank correlation: uncorrected- $\mathrm{p}=0.04, \mathrm{R}=-0.40$ ).

\begin{tabular}{|c|c|c|c|c|c|c|c|}
\hline \multirow[b]{2}{*}{$\begin{array}{l}\text { Anatomical } \\
\text { regions }\end{array}$} & \multirow{2}{*}{\begin{tabular}{|l}
$\begin{array}{l}\text { FWE } \\
\text { corrected p }\end{array}$ \\
(cluster level)
\end{tabular}} & \multirow{2}{*}{$\begin{array}{l}\text { uncorrected p } \\
\text { (cluster level) }\end{array}$} & \multirow[b]{2}{*}{$\begin{array}{l}\text { Cluster } \\
\text { size }\end{array}$} & \multirow{2}{*}{$\begin{array}{l}\text { T-value } \\
\text { (Voxel } \\
\text { level) }\end{array}$} & \multicolumn{3}{|c|}{ MNI coordinates } \\
\hline & & & & & $\mathbf{x}$ & $\mathbf{y}$ & $\mathbf{z}$ \\
\hline \multicolumn{8}{|c|}{ Diagnosis effects (MDD < HS) } \\
\hline \multirow[t]{3}{*}{ Left insula lobe* } & 0.049 & 0.006 & 2060 & 4.40 & -44 & 12 & -7 \\
\hline & & & & 4.25 & -54 & 14 & -3 \\
\hline & & & & 4.21 & -48 & 24 & -25 \\
\hline
\end{tabular}

Table 2. Results of VBM analysis. Abbreviations: FWE = family wise error rate; $\mathrm{HS}=$ healthy subjects; $\mathrm{MDD}=$ Major depression disorders. *See Figure. 


\begin{tabular}{|c|c|c|c|}
\hline & \multirow[b]{2}{*}{ scores $($ mean \pm SD $)$} & \multicolumn{2}{|c|}{$\begin{array}{l}\text { Spearman rank } \\
\text { correlation coefficients }\end{array}$} \\
\hline & & $\mathbf{R}$ & $\mathbf{p}$ \\
\hline Total $(0-52)$ & $21.8 \pm 4.71$ & -0.25 & 0.19 \\
\hline \multicolumn{4}{|l|}{ Subcategory } \\
\hline core $(0-22)$ & $10.3 \pm 2.80$ & -0.26 & 0.18 \\
\hline sleep $(0-4)$ & $3.48 \pm 1.03$ & 0.19 & 0.35 \\
\hline activity $(0-8)$ & $4.00 \pm 1.22$ & -0.23 & 0.25 \\
\hline psychic $(0-8)$ & $2.59 \pm 1.37$ & -0.40 & $0.04 *$ \\
\hline $\begin{array}{l}\text { somatic anxiety } \\
(0-6)\end{array}$ & $3.67 \pm 1.31$ & 0.03 & 0.89 \\
\hline delusion $(0-8)$ & $1.59 \pm 1.03$ & -0.10 & 0.63 \\
\hline
\end{tabular}

Table 3. Relationship between the scores of Hamilton Depression Rating Scale (HAM-D) and GM volume of the left insula lobe. ${ }^{*} \mathrm{p}<0.05$. Ranges of each subcategory score are reported in parentheses.

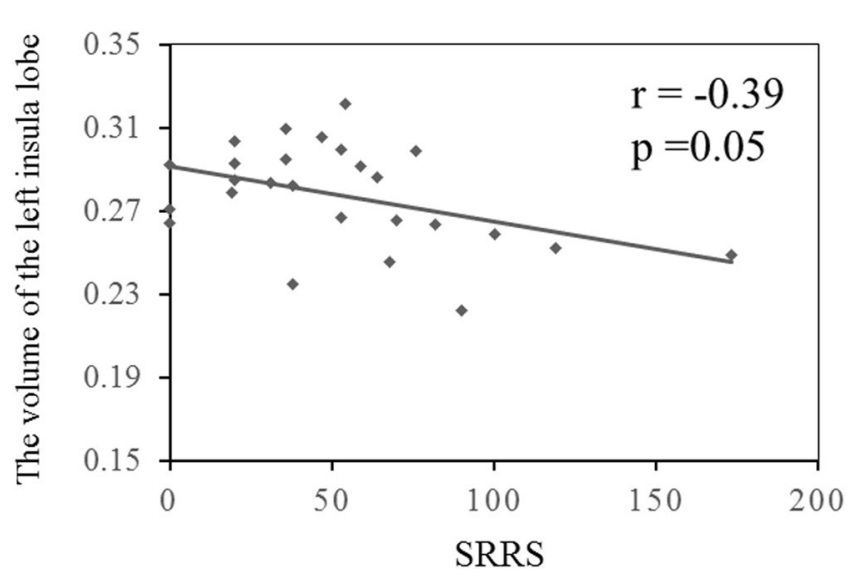

Figure 3. Relationship between the SRRS and the volume of the left insula lobe. The higher SRRS is associated with the volume reduction of the left insula lobe (Spearman rank correlation: uncorrected- $p=0.05, R=-0.39$ ).

insula lobe and the "psychic" score, which consists of the sum of items 9 (agitation including mainly restlessness and psychic agitation) and 10 (psychic anxiety including apprehension, fear, panic, worry, as well as irritability) of the HAM-D, whereas other subcategory scores showed no significant correlation with the left insula lobe volume. Furthermore, the inverse tendency was observed for relationship between the volume of the left insula lobe and the SRRS. This findings strength that dimensional, but not categorical approach for MDD is reasonable. Ohira et al. also reported that Japanese individuals with the s/s genotype of the 5-HTTLPR showed greater blood pressure reactivity to an acute stressor than those with the s/l or $1 / 1$ genotype ${ }^{6}$. Taken together, it is speculated that $s / s$ individuals in Japanese are prone to become depression by psychological stress.

Frodl et al. examined the association between the 5-HTTLPR genotype and GM volumes in MDD patients and showed that patients with the s-allele had significantly smaller subregions within the hippocampus, dorsolateral (DLPFC) and dorsomedial prefrontal cortex (DMPFC) than HS with the same genotype ${ }^{28}$. However, their series for the s-allele included not only the s/s genotype but also the $1 / \mathrm{s}$ genotype. Therefore, no previous studies have performed a simple comparison of the brain volume between MDD patients and the HS strictly regarding the s/s genotype of 5-HTTLPR. To our knowledge, this study provides the first evidence of structural brain alterations in MDD patients with the s/s genotype of 5-HTTLPR. Furthermore, the strength of this study lies in the recruitment of medication naïve MDD patients. Previous studies have shown that the alterations in the brain volume may occur during the course of MDD and after antidepressant treatments ${ }^{29-31}$. Therefore, the morphological changes of the insula might be related to the acute state of MDD, but not the effects caused by antidepressant treatments or the illness chronicity of MDD.

Although the previous study with the relatively large sample size of first-episode medication naïve MDD patients showed widespread GM volume reductions throughout parietal, temporal, limbic regions, and cerebellum $^{32}$, we found only insular cortex change. This result suggests that the volume reduction of the insular lobe may occur prior to other brain regions in MDD patients with the s/s genotype of 5-HTTLPR. The insular lobe is involved in the processing of visceral sensory, visceral motor, vestibular, attention, pain, emotion, verbal, motor information, and input related to music and eating, in addition to gustatory, olfactory, visual, auditory, and tactile data $^{33}$. Recent neuroimaging data, including VBM and fMRI, have revealed that the insular lobe was involved in MDD patients. Our findings are supported by many previous VBM studies that demonstrated a volume reduction of the insular lobe in MDD patients ${ }^{34-36}$; Peng et al. showed a volume reduction of the bilateral insular lobe in first-episode MDD patients ${ }^{36}$. Previous resting-state fMRI studies demonstrated that functional disconnectivity during the resting state has been observed in the insula of MDD patients. Liu et al. demonstrated that, compared 
with HS, MDD patients exhibited significantly decreased regional homogeneity, which measures local connectivity, in the right insula lobe ${ }^{37}$. Iwabuchi et al. found a failure of reciprocal influence between the insula and higher frontal regions in addition to a weakening of influences from sensory regions to the insula lobe ${ }^{38}$. Importantly, Yao et al. showed that a local connectivity reduction in the insula lobe correlated positively with anxiety ${ }^{39}$. This result supports our finding that there was the significant negative correlation between the volume of the insula lobe and the "psychic" score, including agitation and psychic anxiety.

Lesch et al. ${ }^{4}$ reported that the s-allele of 5-HTTLPR was related to anxiety-related traits in healthy Caucasian subjects. Gunthert et al..$^{40}$ and Petersen et al. ${ }^{41}$ also observed that individuals with at least one s-allele who experienced more stressors reported more symptoms of anxiety. The current study showed the negative correlation between the SRRS and the volume of the left insula lobe. Insula lobe is thought to play a key role in mediating homeostatic responses to stress ${ }^{42}$. Our result also may be supported by previous study with healthy subjects which demonstrated that, compared to l-allele, the s-allele appears to be associated with greater reactivity to negative emotional stimuli in the insula ${ }^{43}$. Thus, we speculate that stress events might at least partially affect the insula aberrations. In other words, the integrity of the left insula lobe might be more sensitive to stressful life events than other regions in medication naïve MDD patients with the s/s genotype of the 5-HTTLPR.

Some limitations associated with the present study warrants mention: Because we assessed only subjects with the s/s genotype of 5-HTTLPR, we were unable to examine the interactions between the diagnosis and genotype. In the future, larger studies including MDD patients with the 1/l genotype of 5-HTTLPR will be required to confirm our findings. All MDD patients in this study were right-handers, although roughly $10 \%$ of humans are left-handed. However, we believe that this was unlikely to affect our results, because the previous study found no significant differences in cortical volume between left- and right-handers ${ }^{44}$. MDD is a heterogeneous disorder. However, due to our relatively small sample size, we could not evaluate differences among various MDD subtypes that described in the previous reports ${ }^{10-14}$.

\section{Conclusion}

In a Japanese population with the s/s genotype of 5-HTTLPR, we found a volume reduction of the insula in the early stages of MDD patients compared with HS. This volume reduction also showed a significant positive correlation with the "psychic" score of the HAM-D and the SRRS related to the stressful life events. Our results suggest that, in medication naïve MDD patients with the s/s genotype of the 5-HTTLPR, the insula lobe may play an important role in the "psychic" symptom and the reactivity to stress events. In the future, larger studies including MDD patients with the 1/l genotype of 5-HTTLPR will be required to confirm the effect of genotype of 5-HTTLPR.

\section{References}

1. Chen, H.-T., Clark, M. \& Goldman, D. Quantitative autoradiography of 3H-paroxetine binding sites in rat brain. Journal of pharmacological and toxicological methods 27, 209-216 (1992).

2. Hensler, J. G., Ferry, R. C., Kovachich, G. B. \& Frazer, A. Quantitative autoradiography of the serotonin transporter to assess the distribution of serotonergic projections from the dorsal raphe nucleus. Synapse 17, 1-15 (1994).

3. Collier, D. et al. A novel functional polymorphism within the promoter of the serotonin transporter gene: possible role in susceptibility to affective disorders. Molecular psychiatry 1, 453-460 (1996).

4. Lesch, K.-P., Bengel, D., Heils, A. \& Sabol, S. Z. Association of anxiety-related traits with a polymorphism in the serotonin transporter gene regulatory region. Science 274, 1527 (1996).

5. Bradley, S. L., Dodelzon, K., Sandhu, H. K. \& Philibert, R. A. Relationship of serotonin transporter gene polymorphisms and haplotypes to mRNA transcription. American Journal of Medical Genetics Part B: Neuropsychiatric Genetics 136, 58-61 (2005).

6. Ohira, H. et al. Polymorphism of the serotonin transporter gene modulates brain and physiological responses to acute stress in Japanese men. Stress 12, 533-543 (2009).

7. Gotlib, I. H., Joormann, J., Minor, K. L. \& Hallmayer, J. HPA axis reactivity: a mechanism underlying the associations among 5-HTTLPR, stress, and depression. Biological psychiatry 63, 847-851 (2008).

8. Sharpley, C. F., Palanisamy, S. K., Glyde, N. S., Dillingham, P. W. \& Agnew, L. L. An update on the interaction between the serotonin transporter promoter variant (5-HTTLPR), stress and depression, plus an exploration of non-confirming findings. Behavioural brain research 273, 89-105, doi:10.1016/j.bbr.2014.07.030 (2014).

9. Murakami, F. et al. Anxiety traits associated with a polymorphism in the serotonin transporter gene regulatory region in the Japanese. Journal of human genetics 44, 15-17 (1999).

10. Parker, G. Beyond major depression. Psychological medicine 35, 467-474 (2005).

11. Ostergaard, S. D., Jensen, S. O. \& Bech, P. The heterogeneity of the depressive syndrome: when numbers get serious. Acta psychiatrica Scandinavica 124, 495-496, doi:10.1111/j.1600-0447.2011.01744.x (2011).

12. APA. Diagnostic and statistical manual of mental disorders-V. American Psychiatric Association (2013).

13. Sharpley, C. F. \& Bitsika, V. Differences in neurobiological pathways of four "clinical content" subtypes of depression. Behavioural brain research 256, 368-376, doi:10.1016/j.bbr.2013.08.030 (2013).

14. Sharpley, C. F. \& Bitsika, V. Validity, reliability and prevalence of four 'clinical content' subtypes of depression. Behavioural brain research 259, 9-15, doi:10.1016/j.bbr.2013.10.032 (2014).

15. Serretti, A. et al. Serotonin transporter gene (5-HTTLPR) is not associated with depressive symptomatology in mood disorders. Molecular psychiatry 4, 280-283 (1999).

16. Hayashi, K. et al. COMT Val158Met, but not BDNF Val66Met, is associated with white matter abnormalities of the temporal lobe in patients with first-episode, treatment-naïve major depressive disorder: a diffusion tensor imaging study. Neuropsychiatric disease and treatment 10,1183 (2014).

17. Cardoner, N. et al. Val66Met BDNF genotypes in melancholic depression: effects on brain structure and treatment outcome. Depression and anxiety 30, 225-233, doi:10.1002/da.22025 (2013).

18. Molendijk, M. L. et al. BDNF val66met affects hippocampal volume and emotion-related hippocampal memory activity. Translational psychiatry 2, e74, doi:10.1038/tp.2011.72 (2012).

19. Ide, S. et al. Relationship between a BDNF gene polymorphism and the brain volume in treatment-naive patients with major depressive disorder: A VBM analysis of brain MRI. Psychiatry research 233, 120-124, doi:10.1016/j.pscychresns.2015.05.016 (2015).

20. Pan, C. C. et al. Association analysis of the COMT/MTHFR genes and geriatric depression: an MRI study of the putamen. International journal of geriatric psychiatry 24, 847-855, doi:10.1002/gps.2206 (2009).

21. Watanabe, K. et al. Relationship between the catechol-O-methyl transferase Val108/158Met genotype and brain volume in treatment-naive major depressive disorder: Voxel-based morphometry analysis. Psychiatry research 233, 481-487, doi:10.1016/j. pscychresns.2015.07.024 (2015). 
22. Ueda, I. et al. Relationship between G1287A of the NET Gene Polymorphisms and Brain Volume in Major Depressive Disorder: A Voxel-Based MRI Study. PloS one 11, e0150712, doi:10.1371/journal.pone.0150712 (2016).

23. Jovicich, J. et al. Reliability in multi-site structural MRI studies: effects of gradient non-linearity correction on phantom and human data. Neuroimage 30, 436-443 (2006).

24. Sled, J. G., Zijdenbos, A. P. \& Evans, A. C. A nonparametric method for automatic correction of intensity nonuniformity in MRI data. IEEE Trans Med Imaging 17, 87-97, doi:10.1109/42.668698 (1998).

25. Ashburner, J. Computational anatomy with the SPM software. Magn Reson Imaging 27, 1163-1174, doi:10.1016/j.mri.2009.01.006 (2009).

26. Ashburner, J. A fast diffeomorphic image registration algorithm. Neuroimage 38, 95-113, doi:10.1016/j.neuroimage.2007.07.007 (2007).

27. Holmes, T. H. \& Rahe, R. H. The Social Readjustment Rating Scale. Journal of psychosomatic research 11, 213-218 (1967).

28. Frodl, T. et al. Reduced gray matter brain volumes are associated with variants of the serotonin transporter gene in major depression. Molecular psychiatry 13, 1093-1101 (2008).

29. Malberg, J. E., Eisch, A. J., Nestler, E. J. \& Duman, R. S. Chronic antidepressant treatment increases neurogenesis in adult rat hippocampus. J Neurosci 20, 9104-9110 (2000).

30. Hamilton, J. P., Siemer, M. \& Gotlib, I. H. Amygdala volume in major depressive disorder: a meta-analysis of magnetic resonance imaging studies. Mol Psychiatry 13, 993-1000, doi:10.1038/mp.2008.57 (2008).

31. Zeng, L. L. et al. Antidepressant treatment normalizes white matter volume in patients with major depression. PLoS One 7, e44248, doi:10.1371/journal.pone.0044248 (2012)

32. Shen, Z. et al. Changes of grey matter volume in first-episode drug-naive adult major depressive disorder patients with different age-onset. NeuroImage. Clinical 12, 492-498, doi:10.1016/j.nicl.2016.08.016 (2016).

33. Nagai, M., Kishi, K. \& Kato, S. Insular cortex and neuropsychiatric disorders: a review of recent literature. European Psychiatry 22, 387-394 (2007).

34. Bora, E., Fornito, A., Pantelis, C. \& Yücel, M. Gray matter abnormalities in major depressive disorder: a meta-analysis of voxel based morphometry studies. Journal of affective disorders 138, 9-18 (2012).

35. Sprengelmeyer, R. et al. The insular cortex and the neuroanatomy of major depression. Journal of affective disorders 133, 120-127 (2011).

36. Peng, J. et al. Cerebral and cerebellar gray matter reduction in first-episode patients with major depressive disorder: a voxel-based morphometry study. European journal of radiology 80, 395-399 (2011).

37. Liu, Z. et al. Decreased regional homogeneity in insula and cerebellum: a resting-state fMRI study in patients with major depression and subjects at high risk for major depression. Psychiatry Research: Neuroimaging 182, 211-215 (2010).

38. Iwabuchi, S. J. et al. Alterations in effective connectivity anchored on the insula in major depressive disorder. European Neuropsychopharmacology 24, 1784-1792 (2014).

39. Yao, Z., Wang, L., Lu, Q., Liu, H. \& Teng, G. Regional homogeneity in depression and its relationship with separate depressive symptom clusters: a resting-state fMRI study. Journal of affective disorders 115, 430-438 (2009).

40. Gunthert, K. C. et al. Serotonin transporter gene polymorphism (5-HTTLPR) and anxiety reactivity in daily life: A daily process approach to gene-environment interaction. Psychosomatic medicine 69, 762-768 (2007).

41. Petersen, I. T. et al. Interaction between serotonin transporter polymorphism (5-HTTLPR) and stressful life events in adolescents' trajectories of anxious/depressed symptoms. Developmental psychology 48, 1463 (2012).

42. Paulus, M. P. \& Stein, M. B. An insular view of anxiety. Biological psychiatry 60, 383-387, doi:10.1016/j.biopsych.2006.03.042 (2006).

43. Canli, T. et al. Beyond affect: a role for genetic variation of the serotonin transporter in neural activation during a cognitive attention task. Proceedings of the National Academy of Sciences of the United States of America 102, 12224-12229, doi:10.1073/pnas.0503880102 (2005).

44. Kavaklioglu, T. et al. Structural asymmetries of the human cerebellum in relation to cerebral cortical asymmetries and handedness. Brain structure \& function. doi:10.1007/s00429-016-1295-9 (2016).

45. Allison, T., Puce, A. \& McCarthy, G. Social perception from visual cues: role of the STS region. Trends in cognitive sciences 4 , $267-278(2000)$

\section{Acknowledgements}

This work was supported by a Grant-in-Aid for Scientific Research on Innovative Areas (Comprehensive Brain Science Network) from the Ministry of Education, Science, Sports and Culture of Japan.

\section{Author Contributions}

N.Ig. and S.K. conceived and designed the experiments. N.Ig., K.W., S.I., R.I. and A.K. performed the experiments. N.Ig., K.W. and S.K. analysed the data. N.Ig. composed the manuscript. S.K., O.A., T.K., N.I. and Y.K. provided expertise and edited the manuscript. All authors read the manuscript and are solely and jointly responsible for its content.

\section{Additional Information}

Competing Interests: The authors declare that they have no competing interests.

Publisher's note: Springer Nature remains neutral with regard to jurisdictional claims in published maps and institutional affiliations.

Open Access This article is licensed under a Creative Commons Attribution 4.0 International License, which permits use, sharing, adaptation, distribution and reproduction in any medium or format, as long as you give appropriate credit to the original author(s) and the source, provide a link to the Creative Commons license, and indicate if changes were made. The images or other third party material in this article are included in the article's Creative Commons license, unless indicated otherwise in a credit line to the material. If material is not included in the article's Creative Commons license and your intended use is not permitted by statutory regulation or exceeds the permitted use, you will need to obtain permission directly from the copyright holder. To view a copy of this license, visit http://creativecommons.org/licenses/by/4.0/.

(C) The Author(s) 2017 